\title{
Project-based Language Learning in China: A Literature Review
}

\author{
Sue Wang \\ School of Foreign Studies, Central University of Finance and Economics, Beijing, China
}

\begin{abstract}
Project-based language learning (PBLL) has great potential for language education because PBLL would be able to make learning relevant, personalized, and engaging. This is particularly true under today's circumstances where language learners' are expected to reach the five goals in areas of communication, cultures, comparison, connection and communities as put forward by the ACTFL (www.actfl.org). This paper presents a content analysis of 39 PBLL research studies published between 2002 and 2017 in the English-as-a-Foreign-Language (EFL) context in China. The studies were collected from the two most frequently used Chinese academic journal databases-CNKI (China National Knowledge Infrastructure) and Wanfang Data. This paper presents a synthesis of the PBLL approach as applied in EFL instruction in China, discusses the research results, gaps, and future directions for PBLL research and pedagogy.
\end{abstract}

Index Terms_-project-based language learning, China, EFL, Literature review

\section{INTRODUCTION}

Project-Based Learning (PBL), as a learner-centered approach, was applied to English as a second/foreign language (ESL/EFL) teaching/learning in the 1980s in Europe and in the latter 1990s in North America. Beckett (1999) defined PBLL (Project-Based Language Learning) as a series of individual or group activities that involve language/content learning through planning, researching (empirical and/or document), analyzing and synthesizing data, and reflecting on the process and product orally and/or in writing by comparing, contrasting, and justifying alternatives (p. 4). Since then, numerous studies on PBL as an approach to ESL/EFL learning and instruction have been conducted (see Alan \& Stoller, 2005; Beckett, 2002, 2005; Beckett \& Slater, 2005; Beckett \& Miller, 2006). In China, PBLL studies began to appear in publications in 2002. Since then, PBLL pedagogy and research have attracted an increasing number of scholars, as evidenced by the increasing number of publications.

This paper seeks to address how the PBLL approach is applied in the Chinese EFL context, what the representative PBLL studies have investigated, and the problems and challenges facing PBLL in China. The answers to these questions are important for us to understand the potential benefits of PBLL in China, an important component of the world's language education community and PBLL research in general. To address these questions, this paper presents a synthesis of the PBLL approach as applied to EFL instruction in China between 2002 and 2017, discusses the research results, gaps, and suggests directions for future studies. By examining the challenges of PBLL as presented in the Chinese literature and identifying the future directions of PBL research in the China context, this paper attempts to provide information for the international academic community, of which China is a part, although still at the peripheral. Such a discussion can help inform other peripheral communities in similar contexts.

\section{RESEARCH METHODOLOGY}

\section{A. Data Selection Process and Criteria}

The PBLL studies for this literature review were first collected from the most frequently used CNKI (China National Knowledge Infrastructure), which is a collection of several databases, including academic journals, theses and dissertations, newspapers, and conferences. Another database group, Wanfang Data, was also searched as a supplementary data source. The author manually searched the databases entering the theme 'project learning' confined by subcategory of 'foreign languages and literature'. The time period was set from 2002 through 2017, a span of sixteen years since the first PBLL peer-reviewed article was published in 2002. The theme, project learning, is searched together with its subcategories, such as project-based learning, project instruction, project-based language learning and project-based language teaching. 38 master's theses and two doctoral dissertations were also found since 2003 but this literature review solely focused on published journal articles to avoid possible duplicity, due to the fact that they might be included in the published journal articles.

This preliminary search and subsequent reading of the studies found that in spite of the large number of papers found, the large majority lacked rigor as indicated by their missing literature reviews, methodology, inadequate discussion, and length, which were only 1 or 2 pages (see Zhao, Beckett, \& Wang, 2017, for what constitutes a good research article). The search is then narrowed down to those published in key academic journals as identified by PKU index (the eighth edition of A Guide to Chinese Key Journals, published by Peking University, a nationally recognized index of key 
journals across disciplines) or CSSCI (Chinese Social Sciences Citation Index, another nationally recognized index of key journals published by Nanjing University). Eventually, 39 studies were selected for the current review. Among these, 17 articles were published in the key language education journals and 22 articles published in other key journals, which not only publish English language learning and teaching issues, but also other topics.

\section{B. Coding and Analysis}

After selecting relevant articles, the studies were then classified, based on four categories: empirical studies, topic discussions or theoretical explorations, practice papers, and research reviews as shown in Table 1 below.

TABLE 1

CATEGORIES OF ARTICLES REVIEWED

\begin{tabular}{|l|l|l|}
\hline Articles reviewed & Categories & Number of Articles \\
\hline \multirow{5}{*}{} & Empirical studies & 23 \\
\cline { 2 - 3 } & Topic discussions or theoretical explorations & 10 \\
\cline { 2 - 3 } & Practice papers & 3 \\
\cline { 2 - 3 } & Reviews & 3 \\
\hline Total & & 39 \\
\hline
\end{tabular}

Three review studies were found concerning the PBLL in China. The first, by Zhang (2010), reviewed the thirty-year PBLL research outside China. The author found though PBLL was still at an early stage of experimentation and theoretic exploration, international PBLL researchers' work has attracted more attention in the applied linguistic field. The second, Zhu (2010), discussed PBL in general, with some discussion of PBLL in foreign language teaching and learning. Zhu found that among the 659 studies on PBL (PBL studies across all subject areas from 1998 through 2009), only 10 were language-related, indicating the start of the field. The third, Liu (2011), pointed out the issues of PBLL studies in China, including an uneven distribution of research participants, a lack of systematic studies as well as a lack of depth in the studies. For these reasons, Liu suggested that researchers in this field should produce more rigorous studies in the future so that the research results could better guide the practice. These reviews help us understand the topic of PBLL in general, but an up-date review will help track the latest development in the field and see whether the issues mentioned in Liu (2011) has been addressed.

Besides the review articles, the empirical studies were coded in accordance with the research topics, research questions/problem, research participant groups, methodology, theoretical background or elaboration, and findings. 'Topic discussions or theoretical explorations' refer to articles that discussed or analyzed PBLL, but did not follow the procedure of good empirical research article. 'Practice paper' refers to studies that mainly introduced applications of PBLL in teaching with little or no analysis or discussion. These studies were analyzed based on their study focus and several themes emerged as described in the next section.

\section{FINDINGS AND DISCUSSION}

\section{A. PBLL Studies in China: Quantity and Quality}

A preliminary search showed that there were more than 500 papers published in PBLL and the number on PBLL increased rapidly from 2002-2017. Yet the current literature review finally decided to limit the number to the 39 studies published on PKU Index or CSSCI articles because many of the articles lacked research vigor required. Within the 39 studies in this literature review, there were 9 articles published from 2002 to 2009, and 30 from 2010 to 2017 , an indication of an increased interest. The articles published in the language education journals are in general of higher quality than those published in other key journals as indicated by more empirical studies, better research design and more thorough discussion. The existence of practice papers and topic discussions also indicated the increasing interest in PBLL. At the same time, it should be noted that these articles, except a few, were still only sketchy discussions of PBLL, probably due to their limited length of two or three pages, without enough space to elaborate on the topic.

TABLE 2

CATEGORIES OF ARTICLES REVIEWED

\begin{tabular}{|c|c|c|c|c|}
\hline & & & & \\
\hline & & & & lotal \\
\hline \multirow{4}{*}{$\begin{array}{ll}\text { Language } & \text { key } \\
\text { journals } & \end{array}$} & Empirical studies & 4 & 8 & 12 \\
\hline & $\begin{array}{ll}\text { Topic discussions } & \text { or } \\
\text { theoretical explorations } & \end{array}$ & 2 & 1 & 3 \\
\hline & Practice paper & 1 & 0 & 1 \\
\hline & Review & 0 & 1 & 1 \\
\hline \multirow[t]{4}{*}{ Other key journals } & Empirical studies & 1 & 10 & 11 \\
\hline & $\begin{array}{lll}\text { Topic discussions } & \text { or } \\
\text { theoretical explorations } & \\
\end{array}$ & 1 & 6 & 7 \\
\hline & Practice paper & 0 & 2 & 2 \\
\hline & Review & 0 & 2 & 2 \\
\hline Total & & 9 & 30 & 39 \\
\hline
\end{tabular}


PBLL has been applied in college English teaching and instruction (Gao, 2010) where English is taken as a compulsory course for non-English major students. The literature also shows that this approach has been applied to other more specified scenarios, such as academic English (Li \& Du, 2014), ESP (English for Specific Purposes) such as business English (H. Wang, 2013), content courses, such as linguistics (M. Zhang, 2012), and Chinese culture teaching (Gu \& Ye, 2017). Gao (2010) described the application of PBLL in a group of 96 medical students who learned to conduct a small-scale research project to fulfil partial requirements for their English course. Findings indicated that besides improving the students' motivation and collaboration, PBLL also improved their overall academic literacy and abilities to use language. Gao also pointed out that language use in the project was relevant to the students' foundation in English, and the instructors played a key role in management of the project. M. Zhang (2012) brought PBLL into a linguistics course. Two groups of third-year English majors were selected for the study with one as the control group. The experimental group had both PBLL and traditional lectures, while the control group had only traditional lectures. For this project, the participants selected their topics and questions to explore, outlined their plans and procedures in their problem solution, specified the resources needed, and presented the final results of their projects. The author concluded that the PBLL approach raised learners' intrinsic motivation in linguistics mostly in two components: (1) reaching their goals and (2) achieving emotional satisfaction experience during the process. The experimental group gained a better understanding of linguistic knowledge and were better able to apply this knowledge as shown through the analysis of four kinds of data collected: (1) pre-test and post-test questionnaires, (2) test on linguistics knowledge learned, (3) portfolio which included the student's study plan, process diary and evaluation package, including self-evaluation and group evaluation, and (4) instructor evaluation. For the research population, among the 23 empirical studies reviewed, 18 focused on college students, including English majors and non-English majors. Two studies researched post-graduates (Chen \& Zhao, 2015; Fu, 2009). Only one study (Xu \& Luo, 2012) examined PBLL with middle school students, and one study (Liu, 2013) examined the application of PBLL with vocational students.

\section{PBLL: Learners' Motivation, Collaboration and Autonomy}

Starting with Gu and Zhu (2002), several studies examined the general effects of PBL on language teaching (e.g., Feng \& Zhu, 2003; Gu, 2007; Gu \& Fang, 2003, Huang, 2004). Gu and Zhu (2002) conducted a case study examining a collaborative writing project via the Internet between a group of Chinese college students and their American counterparts. The authors explored the potential of PBLL, when mediated by technology, in motivating Chinese EFL learners by providing authentic writing and explicit purposes, and achieving positive learning results. As an extension, B. Wang (2013) focused more specifically on the features of PBLL that affected learner motivation. Wang researched 167 non-English-major college students in a university in northeastern China to explore the relationship between PBLL and learner motivation. Wang summarized nine features of PBLL that affect learners' motivations in language learning and divided these into primary and secondary factors. If $80 \%$ of the students tested by the three measurement tools (surveys, interviews, and students' own reflections) considered a factor as important after the quantification of the relevant data, this factor would be considered as a primary factor; otherwise, it would be a secondary factor. According to Wang, the primary factors included collaboration, autonomy, accumulation, exploration, and scaffolding, while the secondary factors include authenticity, reflectivity, cross-disciplines, and technology-relevance. Wang recommended that future PBLL users should take full advantage of the primary factors and improve the secondary factors. Shi (2009) found PBLL to be particularly useful in facilitating students' collaborations, while Deng and Wang (2009) found it to be effective in developing students' autonomy skills. Using an experimental approach, Deng and Wang (2009) compared an experimental group of 61 sophomore students with a control group of 60 to determine whether Internet-mediated PBLL could make a difference in the students' autonomous learning. They found PBLL could improve students' autonomous learning in three aspects: attitudes, abilities, and environment. But the authors also noted that within the EFL context, college students still need much of the instructors' scaffolding to develop their autonomy.

\section{PBLL: Learners' Satisfaction, Efforts and Perceptions}

Earlier studies (Feng \& Zhu, 2003; Gu, 2007; Gu \& Fang, 2003; Gu \& Zhu, 2002; Huang, 2004, Li, 2009; Shi, 2009) explored the effects of PBLL. Several more researchers continued this by focusing more on the learners' experiences such as satisfaction, efforts and perceptions. Wang (2012) investigated students' satisfaction of their learning achievement using the PBLL approach. Using a questionnaire and self-reports for 167 college students, Wang pointed out that PBLL helped students meet multiple learning achievements, including language skills, subject content, and twenty-first century skills. Xia and Zhang (2017) researched learners' efforts and factors influencing the efforts during PBLL. They found students invested large amount of time in PBLL though the efforts differ at the different stages and different activities of PBLL. Students indicated engagement which increased with the progress of the projects. The factors that influence their efforts include the difficulties of activities, goals, and affective experience. Another study (Yu, 2017) presented the perceptions of 250 college students towards PBLL in a Chinese university who used New Experiencing English, a textbook based on the PBLL framework. Yu divided students into four groups (A, B, C, and D) based on their general evaluation of PBLL through a six-point scale survey at the end of the course. Students in Group A $(23.3 \%)$ were very positive about PBLL and were categorized as 'active learners' by the author. Group B (40.20\%) were quite positive about PBLL and were categorized as 'adaptable learners'. Those in Group C (28.40\%) were moderately positive about PBL and were categorized as the 'accepted learners'. Group D students $(9.20 \%)$ were categorized as 
'passive learners'. Yu found that the PBLL approach was heralded as beneficial by the students. At the same time, Yu reported that the more active students were more positive while the more passive learners showed a more defensive or resistant attitude towards the PBLL approach. These studies, by investigating deeper into the aspects of PBLL, could help acquire more empirical evidence of the working system for PBLL.

\section{E. PBLL and Language Development Research}

When examining the effectiveness of PBLL, almost all studies reviewed focused on the general macro effects of the approach, such as improving motivation, collaboration, and autonomy in terms of the learning effect with little emphasis on language focus. In the literature reviewed, only one study examined the role of PBLL in the specific language learning process for writing. Yang and Han (2012) conducted an empirical study on college academic English writing taught in China within the context of college English reform. They divided 267 students from ten different colleges into six classes with three classes as the control group and the other three as the experimental group. Participants were required to write an academic paper on an assigned or self-chosen topic, including conducting a literature review, collecting and analyzing data, and writing a research paper. The PBLL approach was utilized for the experimental group, where the process was emphasized and the students were provided more systematic assistance and feedback from the instructors. The project process also included guidance by instructors who organized tutoring sessions, where students presented and discussed their project progress with group members. In contrast, students in the control group were given an assignment at the beginning of the semester and submitted their paper at the end of the semester, when they received feedback. There was no interaction with the instructors regarding their assignments in between. This meant that for the control group, the task was result/product-oriented. The study found that the experimental group outperformed the control group in topic selection, investigation procedures and methods, data analysis, and writing norms and strategies. The linguistic data collected showed that the papers written by the experimental group were longer, used more complex syntax and more accurate vocabulary, and illustrated a better sense of cohesive devices. However, the authors pointed out that the experimental group made more errors in their use of the language. The study concluded that the experimental group had more chances for authentic use of English and improved their abilities for creative exploration as well as academic English writing. Yet, the authors stopped at the discussion of the general benefits of PBLL without furthering the issue of language forms for EFL learners.

\section{F. PBLL: Theoretical Explorations}

Most of the studies reviewed, both practice papers and empirical studies, were practice-oriented rather than theory oriented. Three practice papers included in this review (Huang, 2004; H. Wang, 2013; Zhu \& Zhang, 2011) introduced the application of PBLL in their practice, with a purpose of providing practice examples for PBLL with little or no theoretical discussion. For the 10 topic discussion studies in this literature review, six were introductions of PBLL, summaries of PBLL practice, or explorations of PBLL procedures (e.g. Chen, 2017; Feng \& Zhu, 2003, Xu \& Luo, 2011).

The four theoretical exploration studies were contributed by three scholars who had conducted both empirical studies and theoretical explorations on PBLL. The first of these four theoretical pieces, Gu (2007), investigated how technology can be integrated with PBLL as a pedagogy for language learning. Gu studied the effects of what she termed 'project-based CALL' (computer-aided language learning) and stressed that PBLL, when integrated with technology, could afford authentic, extensive language communication, plenty of language input and output, multi-channel feedback which would benefit thinking and language, and an improvement in motivation, collaboration, and autonomy.

The second of these four theoretical pieces, Zhang (2011), analyzed the micro contexts, features, and influencing factors of PBLL, and put forward a PBLL framework for foreign language teaching in China. This framework takes language, content, abilities, and emotional factors into its core with possible integration with different courses or extension with other abilities.

The third of these four theoretical pieces, W. Zhang (2012), used the term PBLI (project-based language instruction) to distinguish from PBL-based work in his practice and research on PBLL. Zhang identified differences between PBLI and traditional language classes in terms of teacher-student relationships, the content of teaching, teaching methods, and the roles of teachers and students in the Chinese EFL context where the teachers tend to dominate the class. Zhang argued that traditional language teaching in China emphasized language skills training more strongly and lacked the environment and tasks that would enable the students to implement what they learned into practice. Students were expected to improve their comprehensive abilities, critical thinking, and innovation skills, all of which were difficult to do in the traditional way of teaching. By clarifying the key issues in PBLI, Zhang demonstrated the advantages of implementing PBLL as a pedagogy.

The fourth of these four theoretical pieces, Zhang (2015), stressed the need to localize the PBLL approach in China. Zhang suggested an iPBL model (innovation-oriented projected-based learning) and applied it to English-major education in the Chinese EFL context. The iPBL model tends to orient itself to the development of students' innovations through 'course as projects and projects as part of the course' (Zhang, 2015, p. 21, original in Chinese). The author noted that functioning as a platform, the iPBL model features six stages with what he called "milestones"- the key point for each stage - and promotes integrated comprehensive training for students, including critical thinking and innovative training as well as language and other skills for the newer generation. These three scholars' works identified 
three important processes in PBLL studied in China: the integration of PBLL and technology, the analysis of the relationships in PBLL teaching and learning, and the offering of frameworks for future studies.

\section{Challenges, Gaps AND Future Dirctions}

The literature for PBLL in China reviewed here have corroborated the value of PBLL in EFL teaching and learning. The flexibility and feasibility of PBLL to enable students to experience authentic materials and learning by doing makes it a useful approach for language instructors. Researchers in China have applied it to EFL teaching within diverse scenarios, including general college English teaching, EAP, ESP, and vocational teaching. There is a trend in the PBLL research that suggests that studies are moving from a more general exploration to a more specific investigation, and from a majority of college students study groups to a more diverse student body group. As Yu (2017) mentioned in his study that textbooks have been published based on PBLL, indicating a growing acceptance of PBLL in EFL teaching and an increasing interest in PBLL in applied linguistics in China. At the same time, the PBLL research in China are still facing some challenges and there are many areas remain to be explored.

\section{A. Improving the Research Quality of PBLL in China}

As mentioned previously, a large number of the PBLL studies are still in the early stages in terms of academic rigor. Many studies were repetitive, very brief discussions of the topic, or short practice reports. For this reason, only those published in the key journals were selected for this review. Even within such confining terms, there is still a quality variation, particularly between key journals in language education and those in vocational education, an area where the author's preliminary search showed a large quantity of articles but were eventually left out except only a few because of their lack of rigor. More rigorously conducted studies can help the increasing number of PBLL researchers in China make more contribution to the international PBLL research community.

\section{B. Instructor Factor in the PBLL Approach}

The review of the studies shows that the current research of PBLL China mostly still focus on learners. Although studies, such as Gao (2010), Shi (2009), and Zhang (2015) briefly mentioned that instructors played a key role in the application of the project, future studies need to focus more on the roles instructors play when implementing PBLL. Since projects have been proven to be effective for language instruction (Gu \& Zhu, 2002; B. Wang, 2013), it would be an important issue to design projects appropriately when applying it into language teaching. Compared to traditional teaching approaches in which the instructors dominate, PBLL requires a role change of instructors. Studies such as Zhang and Liu (2010) showed the role change of students when PBLL was used, but there is little research on the beliefs or practices of these instructors who are key agents in PBLL pedagogy. Their perceptions and concepts about PBLL and how to design PBLL effectively is worthy of investigation.

\section{Language Development Focus of PBLL}

Almost all the studies reviewed for this paper focused on the relatively macro aspects of PBLL by highlighting motivation, collaboration, and autonomy of the learners. Some of the studies went a step further to explore factors affecting learner motivation or learners' efforts and perceptions. Yet no study so far, except Yang and Han (2012), has focused on the language development issue during PBLL implementation. Even the study by Yang and Han (2012) did not elaborate sufficiently on language development, probably because the project may not have lasted long enough (one semester) to determine noticeable improvement. If we argue the value of PBLL in language teaching and instruction, language acquisition is still an important component even though other aspects, such as motivation and autonomy, cannot be ignored. Future studies need to address this issue of formal language development. With technology, it may be possible for researchers to record or demonstrate language development through the implementation of PBLL.

\section{K-12: An Area Waiting for Exploration}

The current literature of PBLL in the Chinese EFL field is mostly concentrated on higher and vocational education. As mentioned before most of the vocational education papers were excluded because of the lack of research rigor. For the studies reviewed here, 37 of the 39 were published by researchers in universities or co-authored with researchers in universities with university students as their participants. With the exception of Xu and Luo (2012), who explored middle school students, and Chen (2017), who explored PBLL for elementary students, there is little PBLL research on the K-12 language education for a country of about 108 million (Ministry of Education, 2017). The vast majority of Chinese students are required to learn English as a foreign language from at least Grade 3, with most urban students starting English courses from Grade 1 or kindergarten (Yang, 2014). Within the context of dramatic social and economic change in China, education is also experiencing continuous reforms. PBLL, as an effective approach, has great potential for language education in K-12 classrooms.

\section{E. Theory Development}

Theories used in the literature reviewed in this paper are mostly influenced by social constructivism (Gu \& Zhu, 2002; Liu, 2013; Wang, 2010, 2012, Zhang, \& Liu, 2010). Other theories applied include multiple intelligence (Wang, 2010), situational cognition (Zhu \& Zhang, 2011), and social activity theory (Gao, 2010). However, these studies are still 
exploratory and weak on theoretical foundation (Fu, 2009; Shi, 2009; Yang \& Han, 2012). Quite a number of studies even did not provide a section on theories or provide any information about the theories they used. Here there are two problems with theories. One is that many studies did not present the theoretical framework which to a certain extent, decreased the rigor of their research. Another problem is that many of the studies just limit their research scope to the practice introduction without much discussion on the implications or theoretical development (H. Wang, 2013; Zhu \& Zhang, 2011). So far, only two scholars explored the possibility of building localized PBL theories in the Chinese context (M. Zhang, 2011; W. Zhang, 2015). Yet, theory building about PBLL, as an important part of academic research, should be worthy of more attention from the Chinese EFL research communities.

\section{CONCLUSION}

This literature review shows that PBLL studies in China have been growing quickly in terms of quantity, particularly during the past decade. Research quality is improving but many still fall short of vigorous research standards. PBLL as an instruction approach has been applied to a wide range of scenarios and with a variety of populations from primary students to post graduate students. The studies have been focusing on the general effects including motivation, autonomy, collaboration and students' experiences including satisfaction, perceptions and efforts. PBLL studies in China are also found generally more practice-oriented. A few attempts to develop theories and provide localized frameworks for PBLL.

At the same time, it can be seen from the review that PBLL learning and instruction in China is still in its early stage of development. Research quality is improving but still need to be further strengthened. Although the studies are expanding to different populations, they are mostly with college students. K-12 is a virgin land needs to be explored. In terms of the focus of research, the existing studies focused more on the macro-level of the topic with little discussion on the effect of this approach to language development. The studies mostly centered around the students while relatively little is known is investigated about the instructor factor in the PBLL, including their beliefs and attitudes to PBLL, the design and management of projects, specific difficulties or challenges in the application of PBLL in China, and possible solutions to these problems. PBLL as an effective approach would benefit the Chinese EFL learners if it is more widely adopted in the language education of China. More research should be conducted to exert this approach to its greatest potential.

\section{ACKNOWLEDGEMENTS}

This paper is supported by Foreign Language Education Grant of China (\#GWYJYJJ2018B10) and School of Foreign Studies, Central University of Finance and Economics. I would also like to thank Dr. Gulbahar Beckett and Dr. Tammy Slater of Iowa State University for their valuable feedback on the earlier versions of this paper.

\section{REFERENCES}

[1] Alan, B., \& Stoller, F. L. (2005). Maximizing the benefits of project work in foreign language classrooms. English Teaching Forum, 43 (4), 10-21.

[2] Beckett, G. H. (1999) Project-based instruction in a Canadian secondary school's ESL classes: Goals and evaluations. Unpublished doctoral dissertation, University of British Columbia, Vancouver. Canada.

[3] Beckett, G. H. (2002). Teacher and student evaluations of project-based instruction. TESL Canada Journal, 2, 52-66.

[4] Beckett, G. H. (2005). Academic language and literacy socialization through project-based instruction: ESL student perspectives and issues. Journal of Asian Pacific Communication, 15, 191-206.

[5] Beckett, G. H., \& Slater, T. (2005). The project framework: A tool for language, content, and skills integration. ELT Journal, 2 , 108-116.

[6] Beckett, G. H., \& Miller, P. C. (Eds.). (2006). Project based second and foreign language education: Past, present and future. Greenwich, CT: Information Age Publishing.

[7] Chen, Y. (2017). Module-based project learning design strategies and practice. Shanghai Education and Research, 5, 59-61.

[8] Chen, F., \& Zhao, X. (2015). Research projects in the teaching of the post-graduate English teaching. Academic Degrees and Graduate Education, 10, 35-38.

[9] Deng, Y., \& Wang, X. (2009). An empirical study on developing learner autonomy through project-based learning, Foreign Languages and their Teaching, 8, 31-34, 46-47.

[10] Feng, X., \& Zhu, Y. (2003). Research on the project-based learning on the Web, Technology Enhanced Foreign Language Education, 93, 6-9.

[11] Fu, D. (2009). Project-based learning in Authentic Assessment---A case of English teaching of graduate student in MUC, Journal of Research on Education for Ethnic Minorities, 20, 83-87.

[12] Gao, Y. (2010). The application of project-based learning in college English teaching Foreign Language World, 6, 42-56.

[13] Gu, P. (2007). Project-based CALL pedagogy: Theory and practice, Foreign Language World, 2, 2-8.

[14] Gu, P., \& Fang, Y. (2003). Project-based learning with technology: A constructivist view, Foreign Languages and their Teaching, 8, 28-31, 41.

[15] Gu, P., \& Zhu, M. (2002). Online English writing and project-based learning: A case study, Technology Enhanced Foreign Language Education, 88, 3-7. 
[16] Gu, P., \& Zhu, M. (2002). Online English writing and project-based learning: A case study, Foreign Languages and their Teaching, 88, 3-7.

[17] Gu, W., \& Ye, J. (2017). On PBL-centered college English teaching of the Chinese culture: A case study of English teaching of local Chinese culture, Shandong Foreign Language Teaching, 389 (4), 27-36.

[18] Huang, K. (2004). Language teaching reform and quality education: An overview of PBL in German teaching at Tongji University, Foreign Language Teaching Abroad, 1, 6-10.

[19] Li, L. (2009). College English autonomous learning: Project-based learning approach, Education Exploration, 8, $23-24$.

[20] Li, L., \& Du, J. (2014). PBL mode of academic English in discipline-based college English teaching, Foreign Language Education, 35, 55-58.

[21] Liu, F. (2013). Application of PBL in vocational oral English training, China Adult Education, 7, 120-122.

[22] Liu, Y. (2011). PBL research in FLT: Status quo, problems and countermeasures, Journal of Henan University (Social Science), 51, 133-138.

[23] Ministry of Education (2017). Retrieved from http://www.moe.gov.cn/jyb_sjzl/sjzl_fztjgb/201807/t20180719_343508.html, (accessed 21/1/2019).

[24] Shi, X. (2009). A study on project-based college English autonomous learning, Foreign Language Education in China, 2, $16-27$.

[25] Wang, B. (2010). Application of project-based learning to college English teaching in the networked environment, Journal of Northeastern University, 12 (2), 168-172, 188.

[26] Wang, B. (2012). On students' satisfaction at learning achievement in the project-based college English learning model---A study of PBL from the perspective of social constructivism. Journal of Northeastern University, 14 (5), 461-466.

[27] Wang, B. (2013). An analysis of the effects of PBL model on college English learning motivation, Technology Enhanced Foreign Language Education, 149, 37-41, 68.

[28] Wang, H. (2013). An application of PBL into the Business English course, China University Teaching, 8, 52-54.

[29] Xia, S., \& Zhang, W. (2017). Learners' efforts of the project-based language learning. Foreign Languages and their Teaching, $3,78-88$.

[30] Xu, Y., \& Luo, X. (2011). Project-based language learning abroad and its implications for China's English teaching reform. Elementary \& Secondary Schooling Abroad, 4, 58-60, 65.

[31] Xu, Y., \& Luo, X. (2012). Project-based language learning under the background of English comprehensive practice class of junior high schools, Journal of Sichuan Normal University (Social Sciences Edition), 39 (3), 88-93.

[32] Yang, L., \& Han, G. (2012). An empirical study of the PBL approach to academic college English writing, Foreign Language World, 5, 8-16.

[33] Yang, P. (2014). A review of Chinese K-12 foreign language policies, Education Circle, 28, 17-20.

[34] Yu, W. (2017). Students' perceptions of the impacts of project activities in learning New Experiencing English, Foreign Languages in China, 14 (3), 82-91.

[35] Zhang, M. (2011). Post- method and project-based learning framework in foreign language teaching, Journal of Hebei Normal University (Educational Science Edition), 13 (6), 88-92.

[36] Zhang, M. (2012). Project-based learning and the teaching of linguistics, Journal of Hebei Normal University (Educational Science Edition), 14 (8), 89-91.

[37] Zhang, M., \& Liu, Y. (2010). An empirical study on the first-year English majors' role change in project-based learning, Journal of Hebei University of Science and Technology (Social Sciences), 10 (4), 107-112.

[38] Zhang, W. (2010). Three decades of project-based SL/FL learning and instruction: A review of literature, Foreign Languages in China, 7 (2), 68-74.

[39] Zhang, W. (2012). Localized project-based English teaching, China University Teaching 4, 13-16.

[40] Zhang, W. (2015). iPBL: A localized project-based English teaching and learning model, Foreign Languages in China, (12) 2 , $15-23$.

[41] Zhao, J., Beckett, G. H., \& Wang, L. (2017). Evaluating the research quality of education journals in China: Implications for increasing global impact in peripheral countries. Review of Educational Research, 87 (3), 583-618.

[42] Zhu, F. (2010). A study of project teaching approach in China---Also on applicability of project teaching approach to foreign language teaching in China, Theory and Practice of Education, 30, 54-56.

[43] Zhu, L., \& Zhang, J. (2011) Visual simulation technology in the project-based foreign trade English, China Educational Technology, 299, 110-113.

Sue Wang, Ed. D, is currently Associate Professor at School of Foreign Studies, Central University of Finance and Economics in Beijing, P. R. China. She has published books and papers on Chinese EFL students' oral and writing development. Her research interests also include discourse analysis, teacher development and translation studies. 